\title{
Associations of breast-feeding patterns and introduction of solid foods with childhood bone mass: The Generation R Study
}

\author{
Edith H. van den Hooven ${ }^{1}$, Mounira Gharsalli ${ }^{1,2,3}$, Denise H. M. Heppe ${ }^{1,2,3}$, Hein Raat ${ }^{4}$, Albert Hofman ${ }^{1}$, \\ Oscar H. Franco ${ }^{1}$, Fernando Rivadeneira ${ }^{1,5}$ and Vincent W. V. Jaddoe J,2,3* $^{1,3}$ \\ ${ }^{1}$ Department of Epidemiology, Erasmus MC, University Medical Center Rotterdam, PO Box 2040, 3000 CA Rotterdam, \\ The Netherlands \\ ${ }^{2}$ The Generation R Study Group, Erasmus MC, University Medical Center Rotterdam, PO Box 2040, 3000 CA Rotterdam, \\ The Netherlands \\ ${ }^{3}$ Department of Paediatrics, Erasmus MC, University Medical Center Rotterdam, PO Box 2040, 3000 CA Rotterdam, \\ The Netherlands \\ ${ }^{4}$ Department of Public Health, Erasmus MC, University Medical Center Rotterdam, PO Box 2040, 3000 CA Rotterdam, \\ The Netherlands \\ ${ }^{5}$ Department of Internal Medicine, Erasmus MC, University Medical Center Rotterdam, PO Box 2040, 3000 CA Rotterdam, \\ The Netherlands
}

(Submitted 6 March 2015 - Final revision received 2 December 2015 - Accepted 17 December 2015 - First published online 9 February 2016)

\section{Abstract}

Breast-feeding has been associated with later bone health, but results from previous studies are inconsistent. We examined the associations of breast-feeding patterns and timing of introduction of solids with bone mass at the age of 6 years in a prospective cohort study among 4919 children. We collected information about duration and exclusiveness of breast-feeding and timing of introduction of any solids with postnatal questionnaires. A total body dual-energy X-ray absorptiometry scan was performed at 6 years of age, and bone mineral density (BMD), bone mineral content (BMC), area-adjusted BMC (aBMC) and bone area (BA) were analysed. Compared with children who were ever breast-fed, those never breast-fed had lower BMD $\left(-4.62 \mathrm{mg} / \mathrm{cm}^{2} ; 95 \% \mathrm{CI}-8 \cdot 28,-0 \cdot 97\right)$, BMC $(-8.08 \mathrm{~g} ; 95 \% \mathrm{CI}-12 \cdot 45,-3 \cdot 71)$ and BA $\left(-7 \cdot 03 \mathrm{~cm}{ }^{2} ; 95 \% \mathrm{CI}\right.$ $-12.55,-1.52)$ at 6 years of age. Among all breast-fed children, those who were breast-fed non-exclusively in the first 4 months had higher BMD $\left(2.91 \mathrm{mg} / \mathrm{cm}^{2} ; 95 \%\right.$ CI $\left.0.41,5.41\right)$ and aBMC $(3.97 \mathrm{~g} ; 95 \%$ CI $1 \cdot 30,6 \cdot 64)$ and lower BA $\left(-4.45 \mathrm{~cm}^{2} ; 95 \%\right.$ CI $\left.-8 \cdot 28,-0 \cdot 61\right)$ compared with children breast-fed exclusively for at least 4 months. Compared with introduction of solids between 4 and 5 months, introduction $<4$ months was associated with higher BMD and aBMC, whereas introduction between 5 and 6 months was associated with lower aBMC and higher BA. Additional adjustment for infant vitamin D supplementation did not change the results. In conclusion, results from the present study suggest that ever breast-feeding compared with never breast-feeding is associated with higher bone mass in 6-year-old children, but exclusive breastfeeding for 4 months or longer was not positively associated with bone outcomes.

Key words: Breast-feeding: Introduction of solids: Bone mineral density: Childhood: Cohorts

Accretion of bone mass in early life is an important determinant of peak bone mass in adulthood ${ }^{(1,2)}$. Therefore, nutritional exposures in infancy and childhood may affect bone development and the future risk of osteoporosis ${ }^{(3)}$. Human breast milk contains a variety of growth factors, bioactive factors and free amino acids, which may beneficially affect bone development. However, levels of bone-promoting nutrients such as $\mathrm{Ca}$, vitamin $\mathrm{D}$ and $\mathrm{P}$ may be lower as compared with formula milk ${ }^{(4,5)}$. A few previous studies have evaluated the effects of specific infant formulae $v$. breast milk on infant bone health, showing inconsistent results for the impact of breast-feeding on bone mass accretion ${ }^{(6-8)}$. Nevertheless, these studies mainly focused on the comparison of different infant formulae, rather than breast-feeding characteristics (i.e. exclusivity and duration). Other studies investigated the effect of breast-feeding on later bone health in birth cohorts. Some of these studies observed a beneficial effect of breast-feeding on subsequent bone mass ${ }^{(9-11)}$, whereas others found no association $^{(12,13)}$ or an inverse association ${ }^{(14,15)}$. However, these previous studies were of relatively small sample sizes and did

Abbreviations: aBMC, area-adjusted bone mineral content; BA, bone area; BMC, bone mineral content; BMD, bone mineral density; DXA, dual-energy X-ray absorptiometry.

* Corresponding author: V. W. V. Jaddoe, fax +31 107044645 , email v.jaddoe@erasmusmc.nl 
not always have information on potential confounding factors. For example, previous studies did not consider the role of infant vitamin D supplementation in breast-fed infants on childhood bone mass. As vitamin D is necessary for skeletal growth and breast milk contains low levels of vitamin D, it is recommended to provide vitamin $\mathrm{D}$ supplementation to all breast-fed infants ${ }^{(16)}$. Next to breast-feeding, complementary feeding also influences the intake of macronutrients and micronutrients; therefore, timing of introduction of solids may possibly affect bone development as well.

Therefore, we examined the associations of duration and exclusivity of breast-feeding and timing of introduction of solid foods with childhood bone mass at the age of 6 years in a population-based prospective cohort study including 4919 children. In addition, we examined whether the associations between infant feeding and bone mass differed by infant vitamin D supplementation.

\section{Methods}

\section{Study design}

This study was embedded in the Generation R Study, a population-based prospective cohort study from fetal life onwards in the city of Rotterdam, the Netherlands, which has been described in detail previously ${ }^{(17)}$. Pregnant women were enrolled between 2001 and 2005. The study was approved by the local medical ethics committee. Written informed consent was obtained from all participants. The study was conducted according to the guidelines laid down in the Declaration of Helsinki.

\section{Infant feeding assessments}

Information on breast-feeding initiation and continuation was obtained from delivery reports and postnatal questionnaires at the ages of 2, 6 and 12 months, as previously described ${ }^{(17,18)}$. Mothers were asked whether they ever breast-fed their child (yes/no) and at what age they stopped breast-feeding. Mothers were also asked at what age they introduced other types of milk or solid foods using short FFQ at the ages of 6 and 12 months. Among ever breast-fed children, breast-feeding duration was categorised into four groups: $>0-1.9,2-3.9,4-5.9$ and $\geq 6$ months. Exclusiveness of breast-feeding was categorised into the following: non-exclusive in the first 4 months and exclusive for at least 4 months. Non-exclusive breast-feeding indicates infants who received both breast-feeding and formula feeding or solid foods in the first 4 months of life. Exclusive breast-feeding indicated infants who had been breast-fed without any other types of milk, solids or fluids - other than water, in contrast to the definition from the WHO - during the first 4 months of life ${ }^{(18,19)}$. The analyses focused on breastfeeding duration and exclusivity were performed after the exclusion of never breast-fed children. Information on the introduction of any solid foods including fruit/vegetable snacks, dairy products, bread, nuts, meat and fish was obtained from the short FFQ mentioned earlier. The age of introduction of solids was defined as the earliest age at which any solid food was given for the first time, and was categorised into $<4,4-4 \cdot 9$, $5-5.9$ and $\geq 6$ months.

\section{Bone health outcomes}

Bone measurements in childhood were conducted in a dedicated research centre at the Erasmus Medical Center, Rotterdam. The median age of the children at examination was $6 \cdot 0$ years (95\% range $5 \cdot 7-7 \cdot 4$ ). Total body bone mineral density (BMD) was measured using a dual-energy X-ray absorptiometry (DXA) scan (Idxa; General Electrics - Lunar, 2008), using procedures described previously ${ }^{(20)}$. As recommended by the International Society for Clinical Densitometry, for measurement in children, we used total body except head instead of total body BMD as region of interest for all the analyses ${ }^{(21)}$. BMD measured by DXA was expressed as bone mineral content (BMC $(\mathrm{g}))$ per projected bone area $\left(\mathrm{BA}\left(\mathrm{cm}^{2}\right)\right)$. BMC was calculated from BMD using the projected BA. All measures were adjusted for skeletal size using body height or weight as a covariate in the models to correct for artifacts arising from periods of rapid growth ${ }^{(22)}$; this is needed as areal BMD measured on larger bones overestimates true (volumetric) BMD, whereas on smaller bones it can underestimate BMD across individuals $^{(23,24)}$. To further adjust for size effects and to obtain a measure that better approximates the true volumetric BMD, area-adjusted BMC (aBMC) was derived by regressing BMC on $\mathrm{BA}$ and adding the residuals of the regression model to the mean BMC of the study population ${ }^{(23,25)}$.

\section{Covariates}

We registered maternal age at enrolment and collected information on maternal pre-pregnancy weight, educational level, marital status, household income, parity and folic acid supplementation use during pregnancy using a questionnaire at enrolment. Maternal smoking status was assessed in each trimester during pregnancy, and was categorised into never, until pregnancy was known and continued during pregnancy. Maternal height at enrolment was measured without shoes and heavy clothing, and pre-pregnancy BMI $\left(\mathrm{kg} / \mathrm{m}^{2}\right)$ was calculated. We obtained information about child sex, gestational age at birth and birth weight from medical records and hospital registries. Sex- and gestational age-specific standard deviation scores for birth weight were calculated using Swedish reference data ${ }^{(26)}$. Information on child ethnicity was obtained using a questionnaire at enrolment and was defined according to the classification of Statistics Netherlands based on country of birth of the parents ${ }^{(27)}$. We categorised child ethnicity into the following groups: European (Dutch, Turkish, other European, American and Oceanic), African (Moroccan, other African, Antillean, Surinamese-Creole and Cape Verdean) and Asian descent (Indonesian, other Asian and Surinamese-Hindu) descent according to the three largest ancestral groups. Information on working status after pregnancy was derived from postnatal questionnaires. Information on infant vitamin D supplement use (vitamin D alone or multivitamin) was obtained using postnatal questionnaires at the ages of 2, 6 and 12 months, from which a combined variable was created defining any infant vitamin D supplementation (yes/no) in the 1 st year. At the age of 13 months, three dietary patterns were created from dietary intake data as described in detail 
previously ${ }^{(28)}$. These dietary patterns were labelled as follows: 'potatoes, rice and vegetables' pattern, 'refined grains and confectionery' pattern and 'dairy products and whole grains' pattern. Adherence scores to these patterns were calculated for each child. At the 6-year visit, child weight was measured to the nearest $0.2 \mathrm{~kg}$ and height was measured to the nearest $0.1 \mathrm{~cm}$, without shoes and heavy clothing. Measures of total body fat and lean mass (kg) were derived from the DXA scan. Data on participation in sports and duration of television watching, as indicators of physical activity and sedentary behaviour, respectively, were obtained using a questionnaire at 6 years of age.

\section{Statistical analysis}

First, we compared baseline characteristics between the ever and never breast-feeding groups using $t$ tests for continuous variables normally distributed within the two groups, Kruskal-Wallis tests for continuous variables that were non-normally distributed within the two groups and $\chi^{2}$ tests for categorical variables. Second, we assessed the associations of infant feeding patterns (i.e. ever breast-feeding, breast-feeding duration, exclusive breast-feeding and timing of introduction of solids) with BMD, BMC, aBMC and BA using separate multivariable linear regression models. Crude models were adjusted for child sex, ethnicity, age at visit and height at visit. Potential confounders in the multivariable models were selected based on previous literature or a strong association with the outcome, and were kept in the multivariable model in case of $\mathrm{a} \geq 10 \%$ alteration in effect estimates. Following this procedure, multivariable models were additionally adjusted for maternal pre-pregnancy BMI, parity, educational level, smoking status, folic acid supplementation use, working status, household income, child's birth weight $Z$ score, participation in sports and weight at visit. We adjusted for weight at visit by adding lean mass plus fat mass (as measured by DXA) to the model, thereby excluding the contribution of bone mass to the child's weight. Analyses focused on ever breast-feeding and breastfeeding duration, but not on breast-feeding exclusivity, were additionally adjusted for timing of introduction of solids. Analyses focused on timing of introduction of solids were additionally adjusted for breast-feeding duration. To check for collinearity, we examined the variance inflation factors (VIF) and tolerance values in our multivariable models. We performed sensitivity analyses to check whether omitting the additional adjustments for timing of introduction of solids (in models on breast-feeding) and for breast-feeding duration (in models on timing of introduction of solids) changed the results. In addition, we performed sensitivity analyses, in which the associations between infant feeding patterns and bone outcomes were not adjusted for maternal BMI or maternal smoking status, to examine potential mediating effects. We also examined the influence of additional adjustment for maternal age, marital status, child's gestational age at birth, infant vitamin D supplementation, infant dietary patterns and television watching at the age of 6 years. Furthermore, to assess whether the associations between infant feeding and bone mass differed by the child's sex, gestational age at birth, ethnicity or infant vitamin D supplementation, statistical interaction was evaluated by adding the product term of the infant feeding categories and the covariate as an independent variable to the multivariable models. In addition, the interaction effects of duration of breastfeeding and timing of introduction of solids was tested in the multivariable models. In case of a significant interaction term $(P<0.05)$, the associations between infant feeding categories and bone outcomes were analysed separately within categories of the covariate. Tests for trends were performed using categories of breast-feeding duration or timing of introduction of solids as continuous variables in the analyses. To reduce potential bias associated with missing data, we performed multiple imputations of missing covariates, based on the correlation between the variable with missing values and other subject characteristics ${ }^{(29)}$. Missing data on infant feeding patterns or bone outcomes were not imputed. Data were imputed (ten imputations) according to the Fully Conditional Specification method (predictive mean matching), assuming no monotone missing pattern. Analyses were performed in the original data set and in the imputed data sets. As we found similar effect estimates, we present the pooled results after the multiple imputation procedure. All statistical analyses were performed using IBM SPSS version 21 for Windows (IBM Corp.).

\section{Results}

\section{Subject characteristics}

In total, 9749 children were born to mothers enrolled in the Generation R Study. We restricted the current analysis to singleton live-born children - as twins may have different growth and bone development patterns - with available information on infant feeding and bone outcomes at the age of 6 years. In total, 7295 children participated in the pre-school follow-up (Fig. 1). After excluding twins, information on infant feeding was available for 6616 singleton live-born children. Of these, 6054 participated in follow-up measurements at the age of 6 years. DXA scans were performed successfully in 4919 children.

Of all children, 4545 (92\%) had ever been breast-fed, and 1008 children had been exclusively breast-fed for the first 4 months of life. Baseline characteristics of mothers and children stratified by breast-feeding status are presented in Table 1. Compared with mothers who ever breast-fed their children, mothers who never breast-fed their children had a higher BMI and a lower educational level as well as more often smoked during pregnancy. In addition, children who were never breastfed had a lower gestational age at birth and a lower birth weight, were more often of European descent and were less likely to receive vitamin $\mathrm{D}$ supplementation in the 1 st year of life. Bone outcomes at the age of 6 years were comparable between breast-fed and non-breast-fed children, except for BA that was higher in ever breast-fed children.

Information on bone health was available for $74 \%$ of all singleton children with information on breast-feeding. Nonresponse analyses showed that children without bone data on average had a lower birth weight, were more often of nonEuropean descent, were breast-fed for a shorter period and were less often breast-fed exclusively as well as had mothers who were younger, had a lower educational level and lower household 


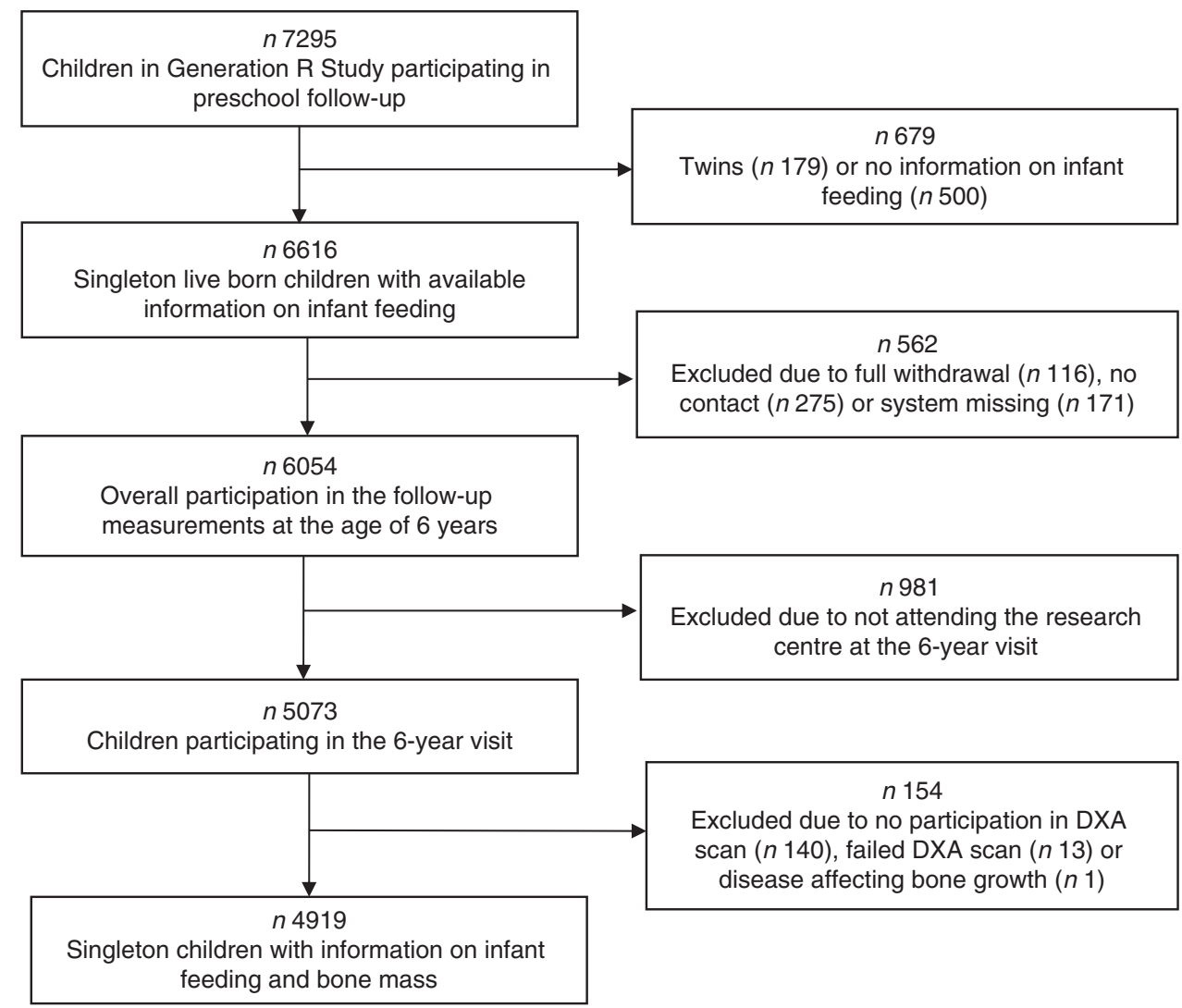

Fig. 1. Population for analysis. DXA, dual-energy X-ray absorptiometry.

income, had a lower BMI, were more often multiparous and were less likely to use folic acid supplements (data not shown).

\section{Infant feeding and childhood bone mass}

Covariate-adjusted associations (adjusted for child sex, ethnicity, age at visit, height at visit, fat mass plus lean mass at visit, maternal pre-pregnancy BMI, parity, educational level, smoking status, folic acid supplementation use, working status, household income, child's birth weight $Z$ score, participation in sports and breast-feeding duration and/or introduction of solids (if applicable)) are presented in Table 2. As compared with children who were ever breast-fed, those who were never breast-fed had a lower BMD $\left(-4.62 \mathrm{mg} / \mathrm{cm}^{2} ; 95 \%\right.$ CI -8.28 , $-0.97)$, BMC (-8.08 g; $95 \% \mathrm{CI}-12.45,-3.71)$ and BA $\left(-7.03 \mathrm{~cm}^{2}\right.$; $95 \%$ CI $-12.55,-1.52$ ) (Table 2 ). Among all breast-fed children, those who were breast-fed non-exclusively in the first 4 months had higher BMD $\left(2.91 \mathrm{mg} / \mathrm{cm}^{2} ; 95 \%\right.$ CI $\left.0.41,5.41\right)$ and aBMC (3.97 g; 95\% CI 1.30, 6.64), but lower BA $\left(-4.45 \mathrm{~cm}^{2}\right.$; $95 \%$ CI $-8 \cdot 28,-0 \cdot 61)$, compared with children who were breast-fed exclusively for at least 4 months. Breast-feeding duration was not associated with bone outcomes. Furthermore, as compared with introduction of solids between 4 and 5 months, introduction before the age of 4 months was associated with a higher BMD $\left(4.10 \mathrm{mg} / \mathrm{cm}^{2} ; 95 \%\right.$ CI 0.46, 7.75) and aBMC (4.06 g; $95 \%$ CI $0 \cdot 18,7 \cdot 94)$, whereas introduction between 5 and 6 months was associated with a lower aBMC $(-3 \cdot 32 ; 95 \% \mathrm{CI}-6 \cdot 50,-0 \cdot 15)$ and higher BA $\left(5.60 \mathrm{~cm}^{2} ; 95 \%\right.$ CI $\left.1.13,10.07\right)$. In addition, introduction at 6 months or older was associated with lower BA $\left(-8.70 \mathrm{~cm}^{2} ; 95 \% \mathrm{CI}-13.85,-3.55\right)$, as compared with introduction between 4 and 5 months.

Crude associations (adjusted for child sex, ethnicity, age at visit, height at visit and fat mass plus lean mass at visit) are presented in online Supplementary Table S1. As compared with the covariate-adjusted associations, we observed smaller effect estimates for the associations between never breast-feeding and bone outcomes and larger effect estimates for the associations between breast-feeding exclusivity and bone outcomes. Furthermore, significant associations were observed for children who were breast-fed for 2-4 months with higher BMD and $\mathrm{aBMC}$, as compared with those who were breast-fed for $\geq 6$ months. These associations changed after adjustment for important maternal and child factors, resulting in the covariate-adjusted associations shown in Table 2 .

\section{Subgroup and sensitivity analyses}

VIF and tolerance values showed no indication of strong collinearity in our statistical models (i.e. all VIF were $<2$ and all tolerance values were $>0 \cdot 4$ ). Further adjustments for maternal age and marital status and child's gestational age at birth, infant dietary patterns and television watching at the age of 6 years did not materially change the effect estimates. After additional adjustment for infant vitamin D supplementation, overall patterns of 
Table 1. Subject characteristics according to categories of breast-feeding ( $n$ 4919)

(Mean values and standard deviations for continuous variables that are normally distributed; medians and $95 \%$ range for continuous variables with a skewed distribution; percentages for categorical variables)

\begin{tabular}{|c|c|c|c|c|c|c|c|}
\hline & \multicolumn{2}{|c|}{ Total group ( $n$ 4919) } & \multicolumn{2}{|c|}{ Never breast-fed ( $n$ 374) } & \multicolumn{2}{|c|}{ Ever breast-fed ( $n$ 4545) } & \multirow[b]{2}{*}{$P^{*}$} \\
\hline & Mean & SD & Mean & SD & Mean & SD & \\
\hline \multicolumn{8}{|l|}{ Maternal characteristics } \\
\hline Age (years) & $31 \cdot 0$ & 4.9 & $31 \cdot 0$ & $4 \cdot 8$ & 31.0 & 4.9 & 0.86 \\
\hline Pre-pregnancy BMl $\left(\mathrm{kg} / \mathrm{m}^{2}\right) \dagger$ & & & \multirow{2}{*}{\multicolumn{2}{|c|}{$23 \cdot 1$}} & \multirow{2}{*}{\multicolumn{2}{|c|}{$22 \cdot 6$}} & 0.01 \\
\hline Median & \multicolumn{2}{|c|}{$22 \cdot 6$} & & & & & \\
\hline $95 \%$ range & \multicolumn{2}{|c|}{$18 \cdot 2-34 \cdot 3$} & \multicolumn{2}{|c|}{$18 \cdot 3-38 \cdot 7$} & \multicolumn{2}{|c|}{$18 \cdot 2-34 \cdot 0$} & \\
\hline Parity (\%) & & & & & & & 0.01 \\
\hline Nulliparous & \multicolumn{2}{|c|}{$56 \cdot 3$} & \multicolumn{2}{|c|}{$48 \cdot 4$} & \multicolumn{2}{|c|}{$56 \cdot 9$} & \\
\hline Multiparous & \multicolumn{2}{|c|}{43.7} & \multicolumn{2}{|c|}{$51 \cdot 6$} & \multicolumn{2}{|c|}{43.1} & \\
\hline Education (\%) & \multirow{2}{*}{\multicolumn{2}{|c|}{$7 \cdot 2$}} & & & & & $<0.001$ \\
\hline Lower & & & \multicolumn{2}{|c|}{6.5} & & & \\
\hline Middle & & & & & & & \\
\hline Higher & & & & & & & \\
\hline Household income (\%) & & & & & & & 0.59 \\
\hline$\leq 2200$ euro/month & & & & & & & \\
\hline$>2200$ euro/month & & & & & & & \\
\hline Smoking during pregnancy (\%) & & & & & & & $<0.001$ \\
\hline Never & & & & & & & \\
\hline Until pregnancy was known & & & & & & & \\
\hline Continued & & & & & & & \\
\hline Folic acid supplement use (\%) & & & & & & & 0.85 \\
\hline Never & & & & & & & \\
\hline Start first 10 weeks & & & & & & & \\
\hline Start periconceptional & & & & & & & \\
\hline Working status after pregnancy (\%) & & & & & & & 0.20 \\
\hline Not working & & & & & & & \\
\hline Started working between $0-4$ months & & & & & & & \\
\hline Started working after 4 months & & & & & & & \\
\hline Infant characteristics & & & & & & & \\
\hline Boys $(\%)$ & & & & & & & 0.99 \\
\hline Gestational age at birth (weeks) $\dagger$ & & & & & & & $<0.001$ \\
\hline Median & & & & & & & \\
\hline $95 \%$ range & 36 & & & & & & \\
\hline Birth weight $(\mathrm{g})$ & 3452 & 539 & 3400 & 557 & 3457 & 537 & 0.05 \\
\hline Ethnicity (\%) & & & & & & & 0.01 \\
\hline European & & & & & & & \\
\hline African & & & & & & & \\
\hline Asian & & & & & & & \\
\hline Any vitamin D supplement use in the 1st year (\%) & & & & & & & $<0.001$ \\
\hline Child characteristics at the 6 -year visit & & & & & & & \\
\hline Age (years) $\dagger$ & & & & & & & 0.75 \\
\hline Median & & & & & & & \\
\hline $95 \%$ range & & & & & & & \\
\hline Height $(\mathrm{cm})$ & $119 \cdot 0$ & $5 \cdot 7$ & 118.6 & 5.7 & $119 \cdot 1$ & 5.6 & $0 \cdot 17$ \\
\hline Weight $(\mathrm{kg}) \dagger$ & & & & & & & 0.37 \\
\hline Median & & & & & & & \\
\hline $95 \%$ range & & & & & & & \\
\hline Participation in sports (\%) & & & & & & & 0.31 \\
\hline Bone mineral density $\left(\mathrm{mg} / \mathrm{cm}^{2}\right)$ & 549 & 48 & 545 & 48 & 549 & 48 & 0.12 \\
\hline Bone mineral content $(\mathrm{g})$ & 518 & 97 & 509 & 98 & 519 & 96 & 0.06 \\
\hline Area-adjusted bone mineral content $(\mathrm{g})$ & 525 & 38 & 524 & 37 & 525 & 38 & 0.81 \\
\hline Bone area $\left(\mathrm{cm}^{2}\right)$ & 939 & 110 & 928 & 112 & 940 & 109 & 0.05 \\
\hline
\end{tabular}

associations were similar, except for the association between never breast-feeding and BMD, which was no longer significant (online Supplementary Table S2). The associations between infant feeding and bone outcomes did not differ by the child's sex, gestational age at birth or infant vitamin D supplementation. Furthermore, no significant interaction effects were observed between breast-feeding duration and timing of introduction of solids in their associations with bone outcomes. In the analyses with BMD and aBMC, we observed (borderline) significant interaction terms between ever breast-feeding and ethnicity ( $P_{\text {interaction }}=0.03$ and 0.07 , respectively). Stratified analyses showed that as compared with ever breast-feeding, never breastfeeding was significantly associated with lower BMD, BMC and BA in European children, but not in African and Asian children (online Supplementary Table S3). In the sensitivity analyses in which models on breast-feeding were not adjusted for timing of 
Table 2. Covariate-adjusted associations of infant feeding with childhood bone mass ( $n$ 4919) ( $\beta$ Coefficients and $95 \%$ confidence intervals) $\dagger$

\begin{tabular}{|c|c|c|c|c|c|c|c|c|}
\hline & \multicolumn{2}{|c|}{$\mathrm{BMD}\left(\mathrm{mg} / \mathrm{cm}^{2}\right)$} & \multicolumn{2}{|c|}{$\mathrm{BMC}(\mathrm{g})$} & \multicolumn{2}{|c|}{$\mathrm{aBMC}(\mathrm{g})$} & \multicolumn{2}{|c|}{$\mathrm{BA}\left(\mathrm{cm}^{2}\right)$} \\
\hline & $\beta$ & $95 \% \mathrm{Cl}$ & $\beta$ & $95 \% \mathrm{Cl}$ & $\beta$ & $95 \% \mathrm{Cl}$ & $\beta$ & $95 \% \mathrm{Cl}$ \\
\hline \multicolumn{9}{|c|}{ Ever breast-feeding ( $n$ 4919) } \\
\hline $\begin{array}{l}\text { Never }(n 374) \\
\text { Ever }(n 4545)\end{array}$ & -4.62 & $\begin{array}{l}-8 \cdot 28,-0.97^{*} \\
\text { Ref. }\end{array}$ & -8.08 & $\begin{array}{l}-12 \cdot 45,-3 \cdot 71^{*} \\
\text { Ref. }\end{array}$ & $-2 \cdot 28$ & $\begin{array}{l}-6 \cdot 22,1 \cdot 66 \\
\text { Ref. }\end{array}$ & -7.03 & $\begin{array}{l}-12.55,-1.52^{*} \\
\text { Ref. }\end{array}$ \\
\hline \multicolumn{9}{|c|}{ Breast-feeding duration ( $n$ 3470) } \\
\hline $0-1.9$ months ( $n$ 976) & 0.74 & $-2 \cdot 39,3 \cdot 88$ & 0.12 & $-3 \cdot 56,3.80$ & 0.59 & $-2 \cdot 84,4 \cdot 01$ & -0.57 & $-5 \cdot 31,4 \cdot 17$ \\
\hline 2-3.9 months ( $n$ 816) & 2.59 & $-0.57,5.76$ & 1.54 & $-2 \cdot 28,5 \cdot 36$ & 2.69 & $-0.72,6.09$ & -1.39 & $-6.22,3.43$ \\
\hline 4-5.9 months ( $n$ 458) & -0.43 & $-4 \cdot 11,3 \cdot 26$ & $-1 \cdot 25$ & $-5 \cdot 77,3 \cdot 27$ & -1.51 & $-5.45,2.43$ & 0.32 & $-5 \cdot 35,5 \cdot 98$ \\
\hline$\geq 6$ months $(n 1220)$ & & Ref. & & Ref. & & Ref. & & Ref. \\
\hline$P_{\text {for trend }}$ & & 0.40 & & 0.73 & & 0.43 & & 0.71 \\
\hline \multicolumn{9}{|c|}{ Exclusive breast-feeding for 4 months ( $n$ 3597) } \\
\hline No $(n$ 2589) & 2.91 & $0.41,5 \cdot 41^{*}$ & 0.30 & $-2 \cdot 75,3 \cdot 35$ & 3.97 & $1 \cdot 30,6 \cdot 64^{*}$ & -4.45 & $-8 \cdot 28,-0 \cdot 61^{*}$ \\
\hline Yes $(n 1008)$ & & Ref. & & Ref. & & Ref. & & Ref. \\
\hline \multicolumn{9}{|c|}{ First introduction of any solids ( $n 3631$ ) } \\
\hline$<4$ months $(n$ 397) & $4 \cdot 10$ & $0.46,7 \cdot 75^{\star}$ & 3.89 & $-2 \cdot 28,4.87$ & 4.06 & $0.18,7.94^{*}$ & -0.21 & $-5 \cdot 70,5 \cdot 28$ \\
\hline 4-4.9 months ( $n$ 2027) & & Ref. & & Ref. & & Ref. & & Ref. \\
\hline $5-5.9$ months $(n 705)$ & -1.97 & $-4.96,1.02$ & 1.29 & $-2 \cdot 28,4 \cdot 87$ & $-3 \cdot 32$ & $-6 \cdot 50,-0 \cdot 15^{\star}$ & $5 \cdot 60$ & $1 \cdot 13,10 \cdot 07^{\star}$ \\
\hline$\geq 6$ months $(n 502)$ & 1.56 & $-1 \cdot 85,2 \cdot 47$ & -3.57 & $-7.67,0.52$ & 3.60 & $-0.03,7 \cdot 23$ & $-8 \cdot 70$ & $-13 \cdot 85,-3 \cdot 55^{\star \star}$ \\
\hline $\bar{P}_{\text {for trend }}$ & & 0.35 & & 0.04 & & 0.79 & & 0.07 \\
\hline
\end{tabular}

$\mathrm{BMD}$, bone mineral density; BMC, bone mineral content; aBMC, area-adjusted BMC; BA, bone area; Ref. referent values.

${ }^{*} P<0.05,{ }^{* *} P<0.01$.

† Values are based on multivariable linear regression models and reflect differences and $95 \% \mathrm{Cl}$ in bone outcomes for categories of breast-feeding and introduction of solid foods. Tests for trend were performed by using categories of breast-feeding or introduction of solids as continuous variables in the analyses. Non-exclusive breast-feeding for 4 months includes partial until 4 months, partial thereafter; and partial until 4 months, not thereafter. Exclusive breast-feeding for 4 months includes exclusive until 6 months; exclusive until 4 months, partial thereafter; and exclusive until 4 months, not thereafter. Models are adjusted for maternal pre-pregnancy BMI, parity, educational level, smoking during pregnancy, folic acid supplementation use, working status, household income, child's sex, birth weight $Z$ score, ethnicity, participation in sports, age at visit, height at visit and fat mass plus lean mass at visit. Models on ever breast-feeding and breast-feeding duration were additionally adjusted for timing of introduction of solids, and models on timing of introduction of solids were additionally adjusted for breast-feeding duration.

introduction of solids, the effect estimates did not change substantially. In addition, patterns of associations were similar if models on timing of introduction of solids were not adjusted for breast-feeding duration (data not shown). Sensitivity analyses in which the associations between infant feeding patterns and bone outcomes were not adjusted for maternal BMI or maternal smoking showed similar patterns of associations (data not shown).

\section{Discussion}

In this large prospective cohort study, we observed that ever breast-feeding as compared with never breast-feeding was positively associated with childhood BMD, BMC and BA. However, the results for duration of breast-feeding, exclusiveness of breast-feeding and timing of introduction of solids did not show a positive association between exclusive breastfeeding for 4 months or longer and subsequent bone outcomes. Specifically, we observed a higher BMD and aBMC in children who were breast-fed non-exclusively in the first 4 months, as compared with exclusively, as well as in children who were introduced to solids before the age of 4 months, as compared with introduction between 4 and 5 months. These associations were independent of infant vitamin D supplementation.

A few previous studies have investigated the effects of breastfeeding on subsequent bone health in participants followed-up from birth. In an Italian study including fifty-seven infants, no differences in bone measures at the age of 12 months were observed between children who were exclusively breast-fed, received formula milk or received both human milk and formula milk ${ }^{(13)}$. In a study among seventy-six American infants, BMC at the age of 12 months was lower in breast-fed infants as compared with formula-fed infants, but this difference did not persist into the 2 nd year of life ${ }^{(14)}$. Similarly, in the Southampton Women's Survey in the UK, duration of breast-feeding was not associated with bone mass at the age of 4 years in 599 children $^{(12)}$. In contrast, duration of exclusive breast-feeding was associated with higher BMD, BMC and BA at the lumbar spine in 109 Danish adolescents at the age of 17 years ${ }^{(10)}$. In addition, in 330 Australian children, those who were breast-fed for $>3$ months had a higher lumbar, hip and total BMD at the age of 8 years compared with those who were formula-fed. These associations were observed in children born at term and not in those born preterm, and there was no evidence for either greater benefit or detriment with longer duration of breast-feeding ${ }^{(9)}$. In the 16-year follow-up of the same cohort, participants who were breast-fed in their 1st month of life had a higher spine and total BMD and a reduced fracture risk, but no associations were observed anymore with breast-feeding for 3 months ${ }^{(11)}$. In an older trial in the UK, preterm infants were randomised to receive human milk or preterm infant formula during the 1st month of life. Follow-up analyses showed that subjects randomised to human milk had higher radial bone mass at the age of 5 years ${ }^{(30)}$, whereas no effect of infant diet on bone mass was observed at the age of $8-12$ years ${ }^{(31)}$. At the age of 20 years, infant diet (i.e. breast milk or formula milk) was not associated with peak bone mass, but the proportion of human milk in the infant diet was positively associated with whole body $\mathrm{BMC}$ and $\mathrm{BA}^{(32)}$. This suggests 
time-specific effects of infant feeding on bone development. Finally, in 158 Finnish adults, shorter breast-feeding duration (i.e. $\leq 3$ months as compared with longer) in infancy was associated with higher BMC and BA of the lumbar spine and higher BMD and BMC of the total body at the age of 32 years in men, but not in women ${ }^{(15)}$. The authors hypothesised that early milk feeding may play an important role in bone mass accrual in males, whereas in females lifestyle and hormonal factors in adulthood, such as contraceptive use, pregnancies and lactation, may influence bone mass to a larger extent than feeding patterns in infancy. In our study of 6-year-old children, associations between breast-feeding and bone mass did not differ between boys and girls. Possibly, these differences, if any, might occur later in life.

The underlying mechanisms linking breast-feeding to childhood bone mass are not well known. Infant formula has higher concentrations of specific nutrients (e.g. Fe, Zn, protein) as compared with breast milk ${ }^{(33)}$, which may explain the inverse associations between breast-feeding and bone mass in some studies. Alternatively, Jones et al. ${ }^{(9,11)}$ proposed that early exposure to breast milk, even for a short period, may programme bone formation during childhood and thereby increase peak bone mass and reduce later fracture risk. In line with this, Fewtrell et $a l^{(32)}$ suggested that the low mineral content of breast milk may programme bone cells to be conservative with minerals later in childhood, resulting in higher bone mass. In addition, the potential beneficial effect of breastfeeding on bone mass could be due to non-nutrient factors such as growth factors and hormones ${ }^{(32)}$, or, alternatively, the observed associations might be explained by residual confounding related to determinants of breast-feeding such as socio-economic status. More research is needed to confirm our findings and to further explore these underlying mechanisms.

In breast-fed children, introduction of solids between 16 and 27 weeks has been suggested to prevent micronutrient deficiencies, including deficiency of Fe, vitamin $\mathrm{A}$ and vitamin $\mathrm{D}^{(33)}$. Therefore, it could be speculated that late introduction of solids might be adversely related to bone development. On the other hand, although results of previous studies are inconsistent, early introduction of solids has been linked to increased risk of developing overweight or obesity ${ }^{(33)}$, which may positively influence bone development indirectly through the mechanical loading by body weight. The literature on the associations between timing of introduction of solids and bone mass accrual in children is scarce. In a trial including 165 infants who were introduced to solid foods at either 3-4 or 6 months, there were no differences in BMC at the age of 6 or 12 months $^{(34)}$. Likewise, a trial including forty-one infants randomised to formula or formula plus cereals reported no differences in BMC after a 10-week period, although the latter group had higher parathyroid hormone concentrations ${ }^{(35)}$, which is known to influence $\mathrm{Ca}$ homoeostasis and bone metabolism.

The recommendations for breast-feeding and introduction of solid foods vary between the WHO and the European Society of Paediatrics, Gastroenterology, Hepatology and Nutrition (ESPGHAN). The WHO recommends exclusive breast-feeding, without other fluids or solids, for the first 6 months of life with the introduction of solid foods thereafter, whereas the ESPGHAN recommends exclusive or full breast-feeding for about 6 months as a desirable goal and that solid foods and liquids other than breast milk or formula should not be introduced before 17 weeks or delayed after 26 weeks of age ${ }^{(33,36,37)}$. For bone development, our results do not support a beneficial effect of exclusive breastfeeding for 4 months or longer. Nevertheless, we must note that the observed associations for duration of breast-feeding and introduction of solids with bone outcomes were not consistent across categories - that is, there was no evidence for a doseresponse relationship. Unfortunately, we had no detailed information on frequency and/or quantity of the solid foods consumed in early life. Further research is needed on the optimal duration of breast-feeding and timing of introduction of solids considering a range of child health and development outcomes, including bone health.

Since the year 2000, daily vitamin D supplementation has been recommended for children up to the age of 4 years (Dutch recommendations) $)^{(38)}$ or for all children (US recommendations $)^{(16)}$. Most of the previous studies did not examine the role of infant vitamin D supplementation in the associations between infant feeding and childhood bone mass. Our results show that the associations between breast-feeding and bone mass changed to some extent, but overall patterns of associations were similar. This might be explained by the fact that the vast majority of children (91\%) in our study received vitamin D supplementation, which could limit the power to detect any differences between those supplemented and not supplemented.

\section{Methodological considerations}

Major strengths of this study are its population-based prospective design from fetal life onwards with a large number of subjects being followed-up, and the availability of detailed information about duration and exclusivity of breast-feeding and childhood bone measurements. In addition, we had extensive information on parental characteristics and potential confounding variables. Nevertheless, the influence of residual confounding due to unmeasured genetic, behaviour and lifestyle factors should still be considered.

We used DXA, a well-validated technique, to assess bone mass accrual in children. However, DXA is a two-dimensional assessment of a three-dimensional structure (3D); therefore areal BMD may be overestimated in larger bones compared with volumetric $\mathrm{BMD}^{(23,24)}$. Further investigations using 3D assessments (e.g. quantitative computerised tomography) are needed to determine whether our findings are arising from differences in skeletal frame size and/or bone geometry. Although most studies evaluating the relationship between infant feeding patterns and bone health have used total body BMD, lumbar spine BMD has also been proposed as a relevant measurement to evaluate paediatric bone health. Unfortunately, in our study, lumbar spine BMD measurements are not available at the age of 6 years. Moreover, we did not collect information on fracture events in our population, as fracture incidence is very low in children of this age without pre-existing health problems. Future studies linking infant feeding to fracture risk at a later age are warranted.

Compared with mothers who ever breast-fed their children, mothers who never breast-fed their children had a higher BMI, 
lower educational level and more often smoked during pregnancy. Moreover, children who were never breast-fed had a lower gestational age at birth and a lower birth weight, were more often of European descent and were less likely to receive vitamin D supplementation in the 1st year of life. The associations between breast-feeding patterns and bone outcomes showed the most distinct effect estimates for never breast-fed children. This may suggest bias by unmeasured confounding factors. Although we adjusted our analyses for a range of maternal and child factors, we cannot exclude the possibility that the observed associations between never breast-feeding and bone outcomes might be due to a cluster of socio-demographic and lifestyle factors associated with breast-feeding.

Breast-feeding information was available in $70 \%$ of the total group of live-born children. Children who participated in the DXA measurement at the age of 6 years were breast-fed for a shorter period and were less often breast-fed exclusively compared with children who did not participate. Selection bias due to selective loss to follow-up is of concern if the associations between infant feeding and bone health differ between those included and not included in the study. Although this seems unlikely, it cannot be excluded.

The observed effects are small and may not reflect clinically relevant differences on an individual level. However, our study provides insight into mechanisms linking infant feeding patterns to bone development during childhood. Future studies are needed to replicate the findings and to evaluate whether the observed differences have consequences for bone health in later life.

\section{Conclusions}

In this prospective cohort study, ever breast-feeding as compared with never breast-feeding was positively associated with bone mass in 6-year-old children, but exclusive breast-feeding for 4 months or longer was not positively associated with childhood bone outcomes. Additional adjustment for infant vitamin D supplementation did not change the observed associations. Further research in other populations is needed to confirm our findings. In addition, future studies should investigate the underlying mechanisms and evaluate whether the observed differences have consequences for bone health in later life.

\section{Acknowledgements}

The Generation R Study is conducted by the Erasmus Medical Center Rotterdam in close collaboration with the School of Law and Faculty of Social Sciences of the Erasmus University Rotterdam; the Municipal Health Service Rotterdam area; the Rotterdam Homecare Foundation; and the Stichting Trombosedienst and Artsenlaboratorium Rijnmond, Rotterdam.

The general design of the Generation $\mathrm{R}$ Study was made possible by financial support from the Erasmus Medical Center, Rotterdam, the Erasmus University Rotterdam, the Dutch Ministry of Health, Welfare and Sport and the Netherlands Organization for Health Research and Development (ZonMw). V. W. V. J. and F. R. received additional grants from the Netherlands Organization for Health Research and Development (VIDI 016.136.361 and VIDI 016.136.367, respectively). E. H. v. d. H. and O. H. F. work at ErasmusAGE, a centre for ageing research across the life course funded by Nestle Nutrition (Nestec Ltd), Metagenics Inc. and AXA. Nestlé Nutrition (Nestec Ltd), Metagenics Inc. and AXA had no role in the design and conduct of the study; collection, management, analysis and interpretation of the data; and preparation, review or approval of the manuscript.

E. H. v. d. H., M. G., D. H. M. H., H. R., A. H., O. H. F., F. R. and V. W. V. J. were involved in the design and planning of the study and data collection; E. H. v. d. H. and M. G. performed statistical analyses; V. W. V. J. provided consultation regarding statistical analyses and interpretation of data; E. H. v. d. H. and M. G. wrote the manuscript; D. H. M. H., H. R., A. H., O. H. F., F. R. and V. W. V. J. provided comments regarding the manuscript; E. H. v. d. H. and V. W. V. J. had primary responsibility for the final content. All the authors read and approved the final version of the paper.

The authors declare that there are no conflicts of interest.

\section{Supplementary material}

For supplementary material/s referred to in this article, please visit http://dx.doi.org/10.1017/S0007114515005462

\section{References}

1. Foley S, Quinn S \& Jones G (2009) Tracking of bone mass from childhood to adolescence and factors that predict deviation from tracking. Bone 44, 752-757.

2. Javaid MK \& Cooper C (2002) Prenatal and childhood influences on osteoporosis. Best Pract Res Clin Endocrinol Metab 16, 349-367.

3. Cooper C, Westlake S, Harvey N, et al. (2006) Review: developmental origins of osteoporotic fracture. Osteoporos Int 17, 337-347.

4. Agostoni C, Carratu B, Boniglia C, et al. (2000) Free amino acid content in standard infant formulas: comparison with human milk. J Am Coll Nutr 19, 434-438.

5. Ballard O \& Morrow AL (2013) Human milk composition: nutrients and bioactive factors. Pediatr Clin North Am 60, 49-74.

6. Kennedy K, Fewtrell MS, Morley R, et al. (1999) Double-blind, randomized trial of a synthetic triacylglycerol in formulafed term infants: effects on stool biochemistry, stool characteristics, and bone mineralization. Am J Clin Nutr 70, 920-927.

7. Litmanovitz I, Davidson K, Eliakim A, et al. (2013) High beta-palmitate formula and bone strength in term infants: a randomized, double-blind, controlled trial. Calcif Tissue Int 92, 35-41.

8. Specker BL, Beck A, Kalkwarf H, et al. (1997) Randomized trial of varying mineral intake on total body bone mineral accretion during the first year of life. Pediatrics 99, E12.

9. Jones G, Riley M \& Dwyer T (2000) Breastfeeding in early life and bone mass in prepubertal children: a longitudinal study. Osteoporos Int 11, 146-152.

10. Molgaard C, Larnkjaer A, Mark AB, et al. (2011) Are early growth and nutrition related to bone health in adolescence? The Copenhagen Cohort Study of infant nutrition and growth. Am J Clin Nutr 94, 6 Suppl., 1865S-1869S. 
11. Jones G, Hynes KL \& Dwyer T (2013) The association between breastfeeding, maternal smoking in utero, and birth weight with bone mass and fractures in adolescents: a 16-year longitudinal study. Osteoporos Int 24, 1605-1611.

12. Harvey NC, Robinson SM, Crozier SR, et al. (2009) Southampton Women's Survey Study G. Breast-feeding and adherence to infant feeding guidelines do not influence bone mass at age 4 years. BrJ Nutr 102, 915-920.

13. Zuccotti G, Vigano A, Cafarelli L, et al. (2011) Longitudinal changes of bone ultrasound measurements in healthy infants during the first year of life: influence of gender and type of feeding. Calcif Tissue Int 89, 312-317.

14. Butte NF, Wong WW, Hopkinson JM, et al. (2000) Infant feeding mode affects early growth and body composition. Pediatrics 106, 1355-1366.

15. Pirila S, Taskinen M, Viljakainen $\mathrm{H}$, et al. (2011) Infant milk feeding influences adult bone health: a prospective study from birth to 32 years. PLOS ONE 6, e19068.

16. Wagner CL \& Greer FR American Academy of Pediatrics Section on Breastfeeding, et al. (2008) Prevention of rickets and vitamin D deficiency in infants, children, and adolescents. Pediatrics 122, 1142-1152.

17. Jaddoe VW, van Duijn CM, Franco OH, et al. (2012) The Generation R Study: design and cohort update 2012. Eur J Epidemiol 27, 739-756.

18. Durmus B, van Rossem L, Duijts L, et al. (2011) Breast-feeding and growth in children until the age of 3 years: the Generation R Study. Br J Nutr 105, 1704-1711.

19. Miliku K, Voortman T, Bakker H, et al. (2015) Infant breastfeeding and kidney function in school-aged children. Am J Kidney Dis 66, 421-428.

20. Heppe DH, Medina-Gomez C, Hofman A, et al. (2013) Maternal first-trimester diet and childhood bone mass: the Generation R Study. Am J Clin Nutr 98, 224-232.

21. Bianchi ML, Baim S, Bishop NJ, et al. (2010) Official positions of the International Society for Clinical Densitometry (ISCD) on DXA evaluation in children and adolescents. Pediatr Nephrol 25, 37-47.

22. Heaney RP (2003) Bone mineral content, not bone mineral density, is the correct bone measure for growth studies. Am J Clin Nutr 78, 350-351; author reply 351-352.

23. Prentice A, Parsons TJ \& Cole TJ (1994) Uncritical use of bone mineral density in absorptiometry may lead to size-related artifacts in the identification of bone mineral determinants. Am J Clin Nutr 60, 837-842.

24. Warner JT, Cowan FJ, Dunstan FD, et al. (1998) Measured and predicted bone mineral content in healthy boys and girls aged 6-18 years: adjustment for body size and puberty. Acta Paediatr 87, 244-249.
25. Macdonald-Wallis C, Tobias JH, Smith GD, et al. (2010) Relation of maternal prepregnancy body mass index with offspring bone mass in childhood: is there evidence for an intrauterine effect? Am J Clin Nutr 92, 872-880.

26. Niklasson A, Ericson A, Fryer JG, et al. (1991) An update of the Swedish reference standards for weight, length and head circumference at birth for given gestational age (1977-1981). Acta Paediatr Scand 80, 756-762.

27. Statistics Netherlands (2004) Migrants in the Netherlands (in Dutch). http://www.cbs.nl/nr/rdonlyres/bd11b2ee-83de47fb-9abc-f8fffe1f3c07/0/2004b52pub.pdf (accessed January 2013).

28. van den Hooven EH, Heppe DH, Kiefte-de Jong JC, et al. (2015) Infant dietary patterns and bone mass in childhood: the Generation R Study. Osteoporos Int 26, 1595-1604.

29. Sterne JA, White IR, Carlin JB, et al. (2009) Multiple imputation for missing data in epidemiological and clinical research: potential and pitfalls. BMJ 338, b2393.

30. Bishop NJ, Dahlenburg SL, Fewtrell MS, et al. (1996) Early diet of preterm infants and bone mineralization at age five years. Acta Paediatr 85, 230-236.

31. Fewtrell MS, Prentice A, Jones SC, et al. (1999) Bone mineralization and turnover in preterm infants at 8-12 years of age: the effect of early diet. J Bone Miner Res 14, 810-820.

32. Fewtrell MS, Williams JE, Singhal A, et al. (2009) Early diet and peak bone mass: 20 year follow-up of a randomized trial of early diet in infants born preterm. Bone 45, 142-149.

33. Agostoni C, Decsi T, Fewtrell M, et al. (2008) Complementary feeding: a commentary by the ESPGHAN Committee on Nutrition. J Pediatr Gastroenterol Nutr 46, 99-110.

34. Mehta KC, Specker BL, Bartholmey S, et al. (1998) Trial on timing of introduction to solids and food type on infant growth. Pediatrics 102, 569-573.

35. Bainbridge RR, Mimouni FB, Landi T, et al. (1996) Effect of rice cereal feedings on bone mineralization and calcium homeostasis in cow milk formula fed infants. J Am Coll Nutr 15, 383-388.

36. World Health Organization (2003) Global Strategy for Infant and Young Child Feeding. Geneva: WHO. http://www. who.int/nutrition/publications/infantfeeding/9241562218/en (accessed November 2014).

37. ESPGHAN Committee on Nutrition, Agostoni C, Braegger C, et al. (2009) Breast-feeding: a commentary by the ESPGHAN committee on nutrition. I Pediatr Gastroenterol Nutr 49 , $112-125$.

38. Health Council of the Netherlands (2008) Towards an adequate intake of vitamin D publication no. 2008/15. http:// www.gezondheidsraad.nl/sites/default/files/200815E.pdf (accessed December 2014). 